\title{
A comparative study of water perfusion catheters and microtip transducer catheters for urethral pressure measurements
}

\author{
Annette Kuhn • Charles W. Nager • Emma Hawkins • \\ Jane Schulz • Stuart L. Stanton
}

Received: 8 May 2006 / Accepted: 25 October 2006 / Published online: 28 November 2006

(C) International Urogynecology Journal 2006

\begin{abstract}
The aim of this study was to compare the maximum urethral closure pressure (MUCP) measures with two different techniques: water perfused catheter and microtip transducer catheters with respect to reproducibility and comparability for urethral pressure measurements. Eighteen women with stress urinary incontinence had repeat static urethral pressure profilometry on a different day using a dual microtip transducer and water perfused catheter (Brown and Wickham). The investigators were blinded to the results of the other. The microtip measurements were taken in the $45^{\circ}$ upright sitting position with the patient at rest at a bladder capacity of $250 \mathrm{ml}$ using an $8 \mathrm{Fr}$ Gaeltec $^{\circledR}$ double microtip transducer withdrawn at $1 \mathrm{~mm} / \mathrm{s}$,
\end{abstract}

This study has been performed without any financial sponsoring by a drug or medical company.

\footnotetext{
A. Kuhn $(\bowtie)$

Department of Urogynaecology, Frauenklinik,

Effingerstr.102, Inselspital,

3011 Bern, Switzerland

e-mail: annette.kuhn@insel.ch

C. W. Nager

Department of Reproductive Medicine,

Division of Urogynecology, University of California,

San Diego, CA, USA

E. Hawkins

Urogynaecology, Haroldswood Hospital,

London, UK

J. Schulz

Urogynecology, Royal Alexandra Hospital,

Edmonton, AB, Canada

\section{S. L. Stanton}

Urogynaecology, 43 Wimpole Street,

London, UK
}

and the transducer was orientated in the three o'clock position. Three different measures were taken for each patient. Three water perfusion measurements were performed with the patient at rest in the $45^{\circ}$ upright position at a bladder capacity of $250 \mathrm{ml}$ using an 8 Fr BARD dual lumen catheter withdrawn at $1 \mathrm{~mm} / \mathrm{s}$. The mean water perfusion MUCP measure was $26.1 \mathrm{~cm} \mathrm{H}_{2} 0$, significantly lower than the mean microtip measure of $35.7 \mathrm{~cm} \mathrm{H}_{2} 0$. The correlation coefficient comparing each water perfusion measurement with the other water perfusion measures in the same patient was excellent, at $0.95(p=0.01)$. Correlation coefficient comparing each microtip measure with the other microtip measure in the same patient was also good, ranging from 0.70 to 0.80 . This study confirms that both water perfusion catheters and microtip transducers have excellent or very good reproducibility with an acceptable intraindividual variation for both methods.

Keywords Microtip transducers .

Urethral pressure measurements - Water perfusion catheters . Stress incontinence

\section{Introduction}

Urethral profilometry is a urodynamic test that is used as a measure of urethral function. Water perfusion catheters with external transducers (water) and electronic catheters with microtip transducers (microtips) are the two technologies in most common clinical use. The water perfusion catheter methodology, first reported by Brown and Wickham in 1969, remains in use in centers that use urodynamic systems with external water transducers. Water perfusion catheters measure the urethral resistance to a slow flow of perfused water through a side hole of a small round 
catheter. This technology meets the description of urethral pressure described by Griffiths and Versi [1] and is recommended by the International Continence Society (ICS) [2]. Water perfusion catheters typically have two or three lumens, are relatively inexpensive, and are disposable. The pressure measuring apertures are connected via pressure tubing to an external pressure transducer. The frequency response of the system is low [3], and therefore, they are not adequate to measure fast cough-like responses or to measure cough pressure transmission ratios.

Microtip catheters have a microtransducer directly attached to the side of the catheter and measure the pressure or force on this surface. These catheters have a rapid frequency response of 2,000 $\mathrm{Hz}$ allowing the recording of rapid pressure changes and cough pressure transmission ratios. They are nondisposable and, in comparison to water perfused catheters, rather expensive. In the urethra, these microtip catheters are not surrounded by fluid, so they are likely measuring the force of the urethral walls on the catheter membrane, and catheter orientation is likely more important than with water perfused catheters [3].

Given the interest and controversy in maximum urethral closure pressure (MUCP) measures, we should know whether the two different measuring systems are comparable and similarly reproducible. Surprisingly, little literature is available on this subject. Wang and Chen [4] found that the two systems were comparably reproducible, but mean measurements were nearly $10 \mathrm{~cm} \mathrm{H}_{2} \mathrm{O}$ higher in the water perfusion group.

The aim of this study was to compare the MUCP measures with two different techniques: (1) water perfusion catheter and (2) microtip catheters with respect to reproducibility and comparability for urethral pressure measurements.

\section{Subjects and methods}

Women enrolled in a study of a new urethral bulking agent in the treatment of stress incontinence were required by study protocol to have static urethral pressure profilometry using a water perfusion catheter. As part of another study on positional variation in urethral pressure measurements, 18 of these women had repeat static urethral pressure profilometry on a different day using a dual microtip transducer [5]. Patients in the latter were recruited from the outpatient department undergoing investigations for urinary stress incontinence.

The study was performed in St. George's Hospital, London.

Both studies received ethical committee approval, and all women gave written informed consent to both studies. All water perfusion catheter measures were performed by the second author $(\mathrm{CWN})$, and all microtip catheter measure- ments were performed by the first author (AK). Both investigators were blinded to the results of the other. Sedia $2000^{\circledR}$ multichannel urodynamic systems were used for the measurements.

The abdominal pressure was measured rectally.

Before the investigations, calibration was performed as requested by the manufacturer.

The external transducer was moved to the upper margin of the symphysis pubis, and zeroing was performed to atmospheric pressure and placed at this level during the procedure as recommended by the ICS. Pves and pura were not equalized during the procedure.

Three water perfusion measurements using sterile lukewarm saline were performed with the patient at rest in the same $45^{\circ}$ upright sitting position at a bladder capacity of $250 \mathrm{ml}$ using an $8 \mathrm{Fr}$ BARD dual lumen catheter withdrawn at $1 \mathrm{~mm} / \mathrm{s}$.

The microtip measurements were taken in the $45^{\circ}$ upright sitting position with the patient at rest at a bladder capacity of $250 \mathrm{ml}$ using a $8 \mathrm{Fr} \mathrm{Gaeltec}{ }^{\circledR}$ double microtip transducer withdrawn at $1 \mathrm{~mm} / \mathrm{s}$, and the transducer was orientated in the three o'clock position. Catheter position was observed during the test to avoid change of orientation. Three different measures were taken for each patient, and patients were randomly assigned to have their urethral pressure profile first in the $45^{\circ}$ upright sitting position or standing.

The same catheter size was used as different gauges may alter the pressure readings [6].

All patients had their water perfusion measurements first.

The catheters were withdrawn with a simultaneous recording of the intravesical and intraurethral pressures, whereof electronic subtraction of these recordings were made. Urethral pressure was recorded as the difference between urethral and intravesical pressure.

The limits of agreement for the two different measurement techniques were calculated as described by Bland and Altman [7]. Reproducibility of the techniques was determined using coefficient of variation [8] and repeatability as appropriate.

The correlation coefficient for the mean water perfusion values and the mean microtip values for each subject were also calculated.

\section{Results}

Our subjects had a mean age of 56 years (range 35-78), median parity of 2 (range $0-4$ ), mean weight of $68 \mathrm{~kg}$ (range $51-90 \mathrm{~kg}$ ) and a mean body mass index of 26 $\mathrm{kg} / \mathrm{m}^{2}$. 
Figure 1 shows a comparison of means with standard deviation, and Fig. 2 a comparison of means with standard error.

Standard error for water perfusion catheter was 2.24 , and for microtip transducer 3.14.

The mean difference between the microtip and the water perfusion methods was $25 \mathrm{~cm} \mathrm{H}_{2} \mathrm{O}$. The limits of agreement for the microtip transducer are from $10 \mathrm{~cm} \mathrm{H}_{2} \mathrm{O}$ below to $60 \mathrm{~cm} \mathrm{H}_{2} \mathrm{O}$ above the water perfusion method.

For MUCP measurements, the within subject variance of the water perfusion method was 7.11 compared with 106.7 for the microtip transducer measurements. Thus, the water perfusion method had a repeatability of $7.4 \mathrm{~cm} \mathrm{H}_{2} \mathrm{O}$, whereas the microtip measurements had a repeatability of $28.6 \mathrm{~cm} \mathrm{H}_{2} \mathrm{O}$.

Table 1 displays the three measures for each technique for all 18 patients and the calculated mean and standard deviation for each patient. The mean water perfusion MUCP measure was $26.1 \mathrm{~cm} \mathrm{H}_{2} 0$, significantly lower than the mean microtip measure of $35.7 \mathrm{~cm} \mathrm{H}_{2} 0$. However, the difference between the MUCPs for each transducer system in each patient may be more important.

The correlation coefficient comparing each water perfusion measurement with the other water perfusion measures in the same patient was excellent, at $0.95(p=0.01)$. The correlation coefficient comparing each microtip measure with the other microtip measure in the same patient was also very good, ranging from 0.70 to 0.80 .

Figure 3 is a scatterplot graphing the mean water perfusion MUCP with the mean microtip MUCP in all 18 patients. The correlation coefficient between these mean values was good, at $0.95(p<0.01)$.

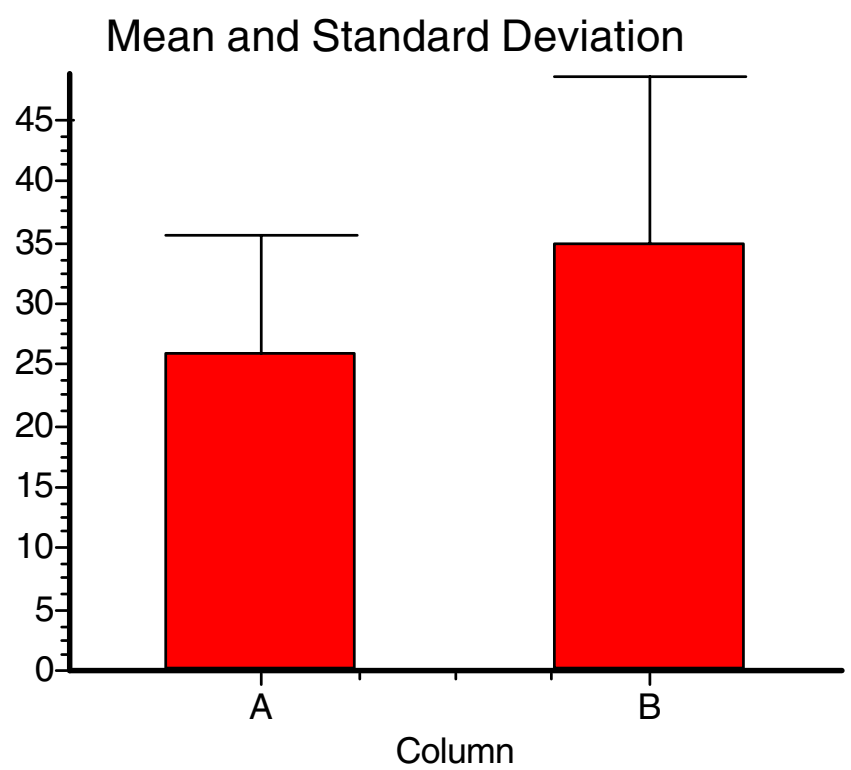

Fig. 1 Mean of water perfusion catheter (column $A$ ) vs microtip catheter (column B)

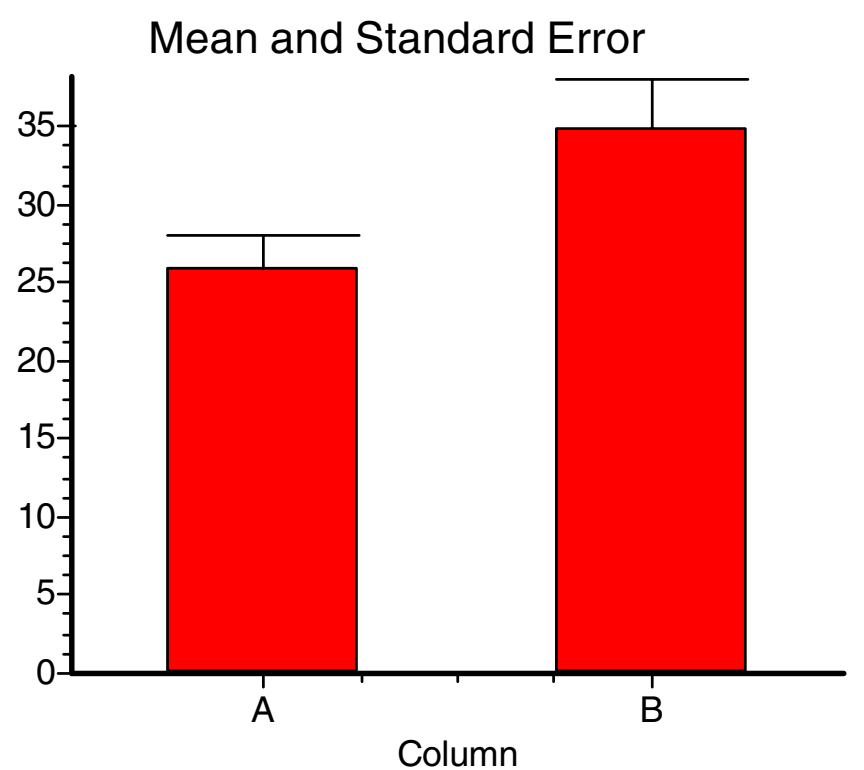

Fig. 2 Standard error of water perfusion (column $A$ ) and microtip transducer (column B)

\section{Discussion}

This study confirms that both water perfusion catheters and microtip transducers have excellent or very good reproducibility with an acceptable intraindividual variation for both methods.

The major finding of our study is that both water perfusion and microtip urethral pressure measurement techniques have very good reproducibility, and intraindividual variation was acceptable for both methods. We do note that the water perfusion technique reproducibility $(r=0.95)$ was better but with no statistical significance $(p=0.06)$ than the microtip reproducibility ( $r=0.7$ to 0.8 ). Due to the small sample size, we cannot draw the conclusion that microtip reproducibility is not as good as water catheters.

The 0.95 correlation coefficient comparing the mean values in each patient for both techniques suggests that these techniques are measuring the same physical and biological phenomena.

The most commonly accepted measure obtained during urethral profilometry is the MUCP. MUCP results may influence recommendations for treatment. The proponents of MUCP testing argue that this test is helpful for: (1) contributing to the differential diagnosis of stress incontinence subtypes intrinsic sphincter deficiency or urethral hypermobility, (2) influencing the choice of therapy, and (3) providing prognostic information for outcome of therapy. When this continuous variable measured by microtip catheters has been used to group patients into categories (MUCP $<20 \mathrm{~cm} / \mathrm{H}_{2} \mathrm{O}$ and MUCP $>20 \mathrm{~cm} \mathrm{H}_{2} 0$ ), most retrospective studies demonstrate that surgical success rates with a modified Burch procedure and sling procedures are 
Table 1 MUCP measures in $\mathrm{cm} /$ water in 18 subjects

\begin{tabular}{|c|c|c|c|c|c|c|c|c|}
\hline $\mathrm{Pt} \#$ & $\mathrm{H}_{2} \mathrm{O} 1$ & $\mathrm{H}_{2} \mathrm{O} 2$ & $\mathrm{H}_{2} \mathrm{O} 3$ & Mtip 1 & Mtip 2 & Mtip 3 & $\mathrm{H}_{2} \mathrm{O} \mathrm{mn}$ (s.d.) & Mtip mn (s.d.) \\
\hline 1 & 21 & 23 & 25 & 30 & 40 & 29 & $23(2)$ & $33(6)$ \\
\hline 2 & 37 & 41 & 30 & 40 & 60 & 32 & $36(6)$ & $44(14)$ \\
\hline 3 & 23 & 24 & 22 & 40 & 38 & 39 & $23(1)$ & $39(1)$ \\
\hline 4 & 40 & 37 & 34 & 61 & 49 & 44 & $37(3)$ & $51(9)$ \\
\hline 5 & 39 & 38 & 37 & 48 & 62 & 56 & $38(1)$ & $55(7)$ \\
\hline 6 & 38 & 32 & 38 & 50 & 45 & 28 & $36(3)$ & $41(12)$ \\
\hline 7 & 17 & 17 & 14 & 25 & 25 & 25 & $16(2)$ & $25(0)$ \\
\hline 8 & 13 & 13 & 13 & 19 & 21 & 17 & $13(0)$ & $19(2)$ \\
\hline 9 & 38 & 34 & 30 & 46 & 50 & 51 & $34(4)$ & $49(3)$ \\
\hline 10 & 47 & 43 & 43 & 53 & 67 & 51 & $44(2)$ & $57(9)$ \\
\hline 11 & 33 & 33 & 33 & 41 & 59 & 41 & $33(0)$ & $47(10)$ \\
\hline 12 & 29 & 25 & 25 & 43 & 43 & 37 & $26(2)$ & $41(3)$ \\
\hline 13 & 14 & 10 & 12 & 21 & 23 & 10 & $12(2)$ & $18(7)$ \\
\hline 14 & 24 & 30 & 27 & 47 & 43 & 12 & $27(3)$ & $34(19)$ \\
\hline 15 & 27 & 21 & 21 & 43 & 27 & 35 & $23(3)$ & $35(8)$ \\
\hline 16 & 13 & 11 & 12 & 20 & 14 & 11 & $12(1)$ & $15(5)$ \\
\hline 17 & 21 & 15 & 15 & 15 & 15 & 24 & $17(3)$ & $18(5)$ \\
\hline 18 & 19 & 18 & 19 & 28 & 12 & 23 & $19(1)$ & $21(8)$ \\
\hline
\end{tabular}

$\mathrm{H}_{2} \mathrm{O}$ heading refers to the three water perfusion measures, and Mtip refers to the three microtip transducer measures in the 18 subjects. $m n$ Mean, s.d. standard deviation

lower in the low MUCP group [9-13]. Regarding this background, it is important to know how comparable and reliable the two test systems are.

Our results are similar to Wang and Chen [4] who also found that the two systems were comparably reproducible. However, their mean measurements were $24.5 \mathrm{~cm} \mathrm{H}_{2} \mathrm{O}$ higher in the water perfusion group, and we found that the mean pressures were nearly $10 \mathrm{~cm}$ higher in the microtip group. However, this study did not blind investigators to the other methods' results; the catheters had different sizes (ten French for water perfused catheters versus six French for microtip catheters), and the catheters' withdrawal speed has not been described. It is possible that the difference in catheter sizes may influence the pressure measurements. Our results are different than some nonblinded investigations, which suggested that microtip transducers give more reliable results [14]; nonblinded investigators may consider the more expensive method as the better and more reliable one.

Possibly, measurements in multiple positions might influence the pressure readings that could influence the study results. However, no data on multiple position measurements of urethral pressure profiles are available.
Fig. 3 Correlation between water perfused and microtip catheter

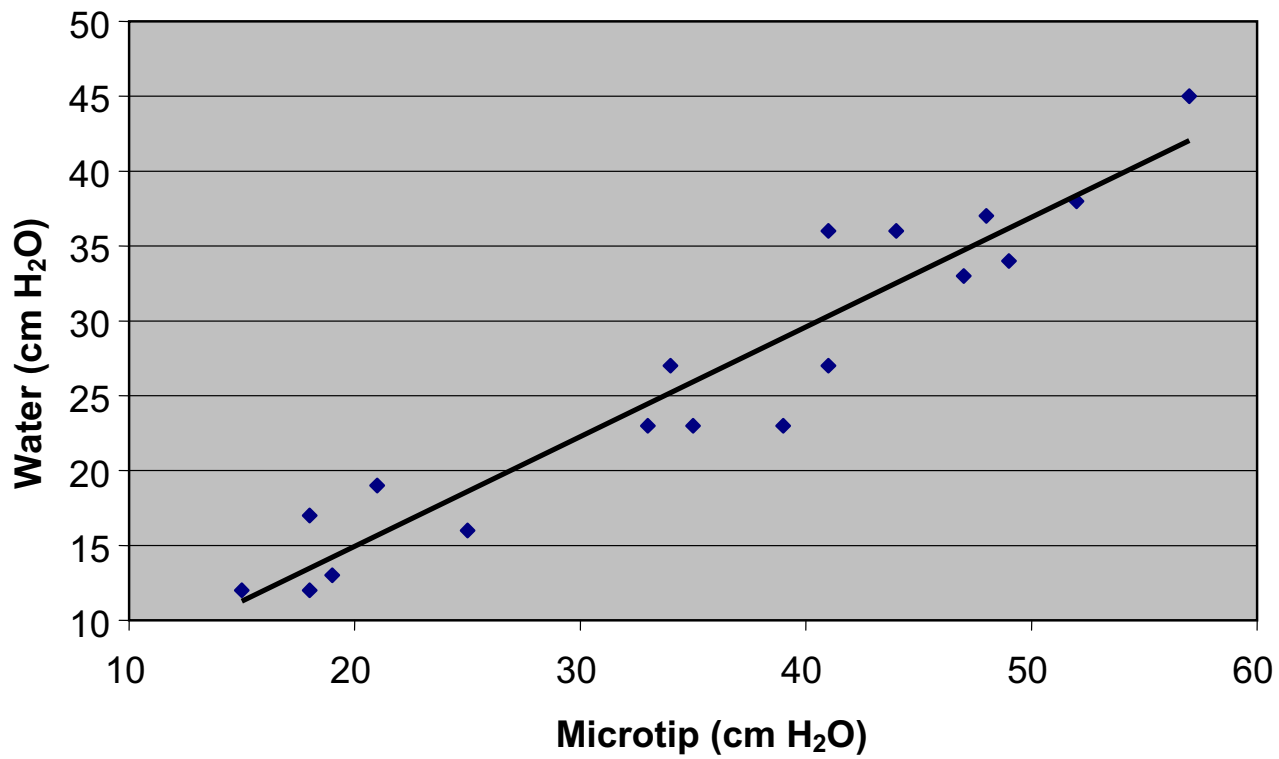


Suburethral tapes have $10 \%$ lower success rates in patients with low pressure urethra [12], which might be important regarding preoperative counseling. More recently, in a series of 70 suburethral transobturator tapes, Davila [15] found significantly higher failure rates with MUCP less than $20 \mathrm{~cm}$ $\mathrm{H}_{2} \mathrm{O}$. Our results would suggest that recommended "cut-off values" would be different depending on the method.

Both systems have their advantages and disadvantages. Disposable water perfusion catheters may be superior in respect to costs and hygienic aspects, as microtip transducers have a potential to accumulate protein deposits that may also affect measurement, yet the perfusion system is more difficult to set up. Microtips allow measures of higher frequency rapid responses [3], such as coughing, but a cleaning process is more arduous, and they are potentially less hygienic due to debris collection at the measurement site. This is speculation only and has not been proven in studies.

A potential limitation of our study is that the microtip transducer and perfusion catheter measurements were made on different days, and it is possible that the differences in pressure were true pressure differences. However, none of the women had any medical or surgical treatment between the two measurements, and the time difference between examinations was less than 1 week. Furthermore, these measures were highly correlated even with the variation in days.

As one author performed all the water perfusion catheter measurements, and the others, all the microtip catheter measurements' bias is potentially introduced into the study. However, the authors were blinded to the results of the other authors' results.

Overall, the value of urethral profilometry remains unclear. MUCP and functional urethral length have never been able to identify stress incontinence. Other urethral function tests, like Valsalva leak point pressure, urethral conductivity, and pressure transmission ratio, fail in this aspect [16].

The 3rd International Consultation of Incontinence (ICI, 2005) summarizes urethral pressure recording in a resting patient as possible but difficult, and stresses that even under standardized methodology there is "a variety of potential artifacts that should be considered critically when interpreting the results" [17].

However, clinically, these tests are widely used as part of urodynamic investigations, and it is worthwhile to look into catheter details when interpreting urethral pressure results and consenting patients.

\section{Conclusions}

In conclusion, water perfusion catheters and microtip transducers are both reliable measures with little intraindividual variation. Water perfusion systems are at least as reliable as microtip transducers.
The major finding of our study is that both water perfusion and microtip urethral pressure measurement techniques have very good reproducibility and the intraindividual variation was acceptable for both methods. This could possibly mean that repeat measurements are not really necessary in clinical practice.

Acknowledgement We thoroughly thank Emily S. Lukacz for her support with the statistical analysis.

\section{References}

1. Griffiths DJ, Versi E (1996) Urethral function. Curr Opin Obstet Gynecol 8(5):372-375

2. Lose G, Griffiths DJ, Hosker G, Kulseng-Hanssen S, Perucchini D, Schafer W, Thind P, Versi E (2002) Standardization subcommittee, International continence society standardisation of urethral pressure measurement: report form the standardization sub-committee of the International continence society. Neurourol Urodyn 21(3):258-260

3. Hilton P, Stanton SL (1986) Urethral pressure measurements by microtip transducer: the results in symptom free women and in those with genuine stress incontinence. Br J Obstet Gynaecol 90:919-934

4. Wang AC, Chen MC (2002) A comparison of urethral pressure profilometry using microtip and double-lumen perfusion catheters in women with genuine stress incontinence. BJOG 109(3):322326

5. Dörflinger A, Gorton E, Stanton S, Dreher E (2002) Urethral pressure profile: is it affected by position? Neurourol Urodyn 22:1-7

6. Bump RC, Elser DM, Theofrastus JP, McClish DK (1995) Valsalva leak point pressures in women with genuine stress incontinence: reproducibility, effect on catheter calibre and correlation with other measures of urethral resistance. Am J Obstet Gynecol 173(2):551-557

7. Bland JM, Altman DG (1986) Statistical methods for assessing agreement between two methods of clinical measurement. Lancet $1: 308-310$

8. Bland JM, Altman DG (1996) Measurement error proportional to the mean. BMJ 313:106

9. Sand PK, Bowen LW, Panganiban R, Ostergard DR (1987) The low pressure urethra as a factor in failed retropubic urethropexy. Obstet Gynecol 69:399-402

10. Bergman A, Koonings PP, Ballard CA (1989) Proposed management of low urethral pressure type of genuine stress urinary incontinence. Gynecol Obstet Investig 27:155-159

11. Koonings PP, Bergman A, Ballard CA (1990) Low urethral pressure and stress urinary incontinence in women: risk factor for failed retropubic surgical procedure. Urology 36:245-248

12. Richardson DA, Ramahi A, Chalas E (1991) Surgical management of stress incontinence in patients with low urethral pressure. Gynecol Obstet Investig 31:106-109

13. Maher CF, Dwyer PL, Carey MP, Moran PA (1999) Colposuspension or sling for low urethral pressure stress incontinence? Int Urogynecol J Pelvic Floor Dysfunct 10:384-389

14. Hilton P (1982) The urethral pressure profile at rest: an analysis of variance. Neurourol Urodyn 1:303-311

15. Davila W (2005) Abstract at the AUGS Meeting, Copenhagen

16. Kuhn A, Kuhn P, Stanton SL (2005) How valuable are urethral function tests? Geburtshilfe Frauenheilkd 65:669-673

17. 3rd International Consultation of Incontinence, 2005, Mechanisms of continence p 593-598 